Research Article

\title{
Collaborative Vehicle Routing Problem in the Urban Ring Logistics Network under the COVID-19 Epidemic
}

\author{
Wenjia Zheng $\mathbb{D}^{1}{ }^{1}$ Zhongyu Wang, ${ }^{1}$ and Liucheng Sun ${ }^{2}$ \\ ${ }^{1}$ College of Transport and Communication, Shanghai Maritime University, Shanghai, China \\ ${ }^{2}$ Alibaba Group, Hangzhou, China \\ Correspondence should be addressed to Wenjia Zheng; cqhbbd_2000@163.com
}

Received 11 January 2021; Accepted 11 November 2021; Published 21 December 2021

Academic Editor: Lei Liu

Copyright ( 92021 Wenjia Zheng et al. This is an open access article distributed under the Creative Commons Attribution License, which permits unrestricted use, distribution, and reproduction in any medium, provided the original work is properly cited.

This paper explored the problem of collaborative vehicle routing in the urban ring logistics network (Co-VRP-URLN) during the COVID-19 epidemic. According to the characteristics of urban distribution and the restriction of traffic all over China during this period, this study mainly considers a common distribution mode of order exchange through the outer ring of the city and then solves the vehicle routing problem of distribution, which belongs to a special multidepot vehicle routing problem with time windows. According to the definition of the problem, the corresponding mixed-integer programming problem of multicommodity flow is established, and the variable neighborhood search algorithm is designed in detail to solve it. The effectiveness of the algorithm is verified by a standard example, and the benefits of joint distribution are revealed through the improved standard example. At last, the influence of different distribution centers is compared. The results show that this model can significantly improve the distribution efficiency within the city under the restriction of traffic.

\section{Introduction}

This research mainly focuses on a problem of centralized planning of vehicle routes for urban joint distribution, whose aim is to evaluate a potential urban joint distribution model under the COVID-19 epidemic. In the urban distribution system, the distribution center (DC) is usually located in the suburb of the city, while customers are all over the city. After the goods enter the city, they will be sent to the distribution center for temporary storage or combination. From the perspective of means of transport, although large trucks are relatively economic means of transport, due to the limitations of urban traffic laws and regulations under the COVID-19 epidemic, they generally do not directly carry out door-to-door distribution services but unload the goods in the distribution center. Then the small- and medium-sized distribution vehicles carry out the final urban distribution services.

For carrier who performs the last step, what it needs to do is to complete the distribution efficiently. Specifically speaking, a team is responsible for distributing the goods and organizing appropriate driving routes under the different number of goods needs of different customers. The goal is to meet the needs of customers and achieve such goals as the shortest distance, the least cost, and the least time consumption under a certain constraint, which is known as vehicle routing problem (VRP).

However, compared with other transportation activities, the profit of urban "last mile" distribution is lower, so carriers are more eager to find ways to further reduce costs. Under the premise of satisfying customer service level, the joint distribution is one of the most potent ways. The carriers participating in the joint distribution can reduce the number of vehicles used and the distance traveled by means of order exchange and consolidation and vehicle sharing, so as to reduce costs and obtain higher benefits. On the other hand, joint distribution will also bring social benefits such as traffic congestion relief and pollution reduction as well as regulations under the COVID-19 epidemic.

In urban areas, a new distribution method based on the ring network is emerging. Combined with the joint distribution, a new kind of vehicle routing problem of urban joint 
distribution is born. Different from the traditional vehicle routing problem, carriers can exchange orders through the urban "outer ring" road using heavy trucks and then distribute them. Figure 1 shows a simple example. It can be seen that, due to the geographical dispersion of the distribution center, through the exchange of orders on the outskirts of the city (some public customers have been effectively merged), the distance between customers and the corresponding goods distribution starting point is shortened, and the passing of distribution vehicles in the city is reduced. At the same time, the number of vehicles used may be reduced, resulting in certain economic and social benefits.

The difficulty of this paper is to study the model and algorithm of this kind of vehicle routing problem. In the next research, the authors will make efforts in the following aspects: (1) give a formal problem description and general mathematical model, (2) briefly introduce the variable neighborhood search algorithm to solve the model, (3) design the specific details of the algorithm, and (4) verify the feasibility of this study by numerical experiments.

\section{Literature Review}

The main contributions of the current literature to the research of joint distribution vehicle routing problem under centralized planning are as follows:

(1) Put forward the joint distribution mode and build a new distribution network.

(2) Establish corresponding model with special constraints and design the algorithm to solve the problem. The research results can guide the implementation of joint distribution to a certain extent and achieve the purpose of evaluating the effect of joint distribution mode. Among them, according to the actual scene of joint distribution, it can be divided into two basic types according to the transportation task form (mainly refers to the different cargo loading and unloading places): one-to-one transportation mode and one-many-one transportation mode. Each type includes the corresponding joint distribution mode and the corresponding vehicle routing problem, which are described in detail below.

2.1. One-to-One Mode. One-to-one means that each batch of goods will be given the starting point (loading point) and terminal point (unloading point). The distribution demand appears in pairs, which can be divided into two subcategories.

\subsubsection{Vehicles Can Only Serve One Batch of Goods at One} Time. In transportation of truck load, Liu et al. [1] proposed a multidepot arc path problem with capacity constraints. The objective function is to minimize the distance of empty vehicles, and a two-stage greedy algorithm is designed to solve large-scale practical problems. Hernandez and Peeta [2] considered a joint distribution problem in which the demand is determined but the transportation capacity of the alliance is time-dependent. A minimum cost flow model is established and a branch pruning algorithm is designed. Speranza et al. [3] studied a kind of arc path problem without capacity limitation under joint distribution, and some customers only wanted to be served by the designated transportation company. According to these conditions, the authors established an integer linear programming model and designed a branch cutting algorithm to solve the problem. Ergun et al. [4] and Kuyzu [5] defined the shippers' cooperation problem and established a model of arc covering problem and pointed out that the most basic arc covering model can be solved by polynomial time. After extending the common arc covering problem with some special agreements of partners, the problem becomes NPhard problem. The algorithm uses column generation and branch and bound to solve the problem accurately.

2.1.2. The Vehicle Can Load More than One Batch of Goods at One Time. For such problems, Dai and Chen [6, 7], respectively, designed Lagrange relaxation algorithm and Benders Decomposition algorithm to solve them. Weng and $\mathrm{Xu}$ [8], respectively, designed two heuristic algorithms based on Lagrange relaxation and Benders Decomposition for a class of multihub arc path problem with distance constraints. Lin [9] considered a new type of joint distribution, in which vehicles were allowed to exchange goods in the middle of the way and be used repeatedly. A constructive heuristic algorithm based on insertion operator was designed.

2.2. One-Many-One Mode. One-many-one means that the loading points of goods are all at the starting point of vehicles (warehouse or yard), while other points are unloading points. This kind of mode is the mainstream way of urban distribution, such as express delivery and supermarket distribution. Compared with one-to-one transportation, there are more joint distribution modes under this mode of transportation, mainly through vehicle sharing or order sharing.

In the evaluation of this kind of joint distribution mode, scholars found that joint distribution reflects good flexibility under the influence of dynamic environment. When the external environment (such as the volume of distribution and delivery) fluctuates, the waste of resources can be effectively avoided through cooperation. Sprenger and Münch [10] simulated a case of a food market in Germany, in which a public distribution center was established to form an important node of the joint distribution network. Then they built a multilevel distribution network of factory-distribution center-customers. In this paper, a large vehicle routing problem model with time windows considering capacity constraints, order delivery, maximum operation time, and outsourcing is established. The model is decomposed into several VRP submodels and ant colony algorithm is designed to solve it. Finally, the method is tested by discrete event simulation, and the results show that joint distribution has good performance under random and dynamic conditions. Quintero-Araujo et al. [11] also discussed the revenue from 
joint distribution in a supply chain with stochastic demand and found that the revenue was increased by $7.3 \%$. Yilmazb [12] established a Markov decision process model to evaluate the operation of small-scale shippers' alliance under uncertain environment and pointed out that although joint distribution can generally bring considerable benefits, timely benefit distribution will greatly affect the stability of the alliance.

The joint distribution can bring economic and social benefits, such as reducing road congestion, noise pollution, and exhaust pollution. Therefore, the government departments also actively support the cooperation of transportation enterprises, especially in the level of intracity distribution. Pérez-Bernabeu et al. [13] compared the results of multidepot vehicle routing problem after joint distribution with those of common vehicle routing problem before joint distribution, which showed that carbon emissions would be significantly reduced. Soysal et al. [14] established the inventory routing problem (IRP) model in the joint distribution environment. The model considered the shelf life, energy use (carbon emissions), and demand uncertainty of goods. The results show that the cost reduction is between $4 \%$ and $24 \%$, and the carbon emission is reduced by about $8 \%-33 \%$. For cooperative IRP problems, Fardi et al. [15] used robust optimization methods to study similar problems.

\section{Problem Description and Mathematical Model}

Under this background, the vehicle routing problem of joint distribution of urban ring network to be studied (collaborative vehicle routing problem in the urban ring logistics network, Co-VRP-URLN) is described. In this problem, there are several carriers whose operating vehicles are ready to serve the same urban area in each distribution center (CDC). Each carrier obtains a batch of order delivery requirements from its own shipper. At the beginning of a delivery cycle, these goods have been stored in their own distribution center. Each order corresponds to one customer, and the revenue of the order, geographical location, demand for goods, service time, and service time window (hard) are given. When they distribute separately, the costs are vehicle fixed cost and vehicle driving cost. In order to reduce costs, carriers form a joint distribution alliance and share their order information: carriers can deliver some orders to partners for distribution. Because the research of this paper is based on the ring distribution network of city, the goods in the distribution center can be redistributed and delivered through the flow of urban "outer ring." The optimization goal of the decision center is to reallocate the orders and the decision of vehicle path, which achieves the minimum cost or the maximum revenue. The constraints are the following:

(1) Vehicle capacity restriction;

(2) The vehicle starting from the distribution center and returning to the distribution center;

(3) Time window constraint (waiting if early);
(4) Each customer being served once and only once. Besides, consider the following:

(1) Assuming that the goods transfer process is completed by the third-party transport company before the start of a distribution cycle (such as at night) or directly unloaded to other distribution centers after entering the city through heavy-duty vehicles, the cost of this additional operation (called repositioning) is called transfer cost. Compared with the urban distribution vehicle, the transportation vehicle load in this process is larger and cheaper. For the convenience of later research, it is simply assumed that the cost is directly proportional to the transportation volume and distance.

(2) There is no limit on the number of vehicles, but each vehicle has fixed cost.

(3) Assume that the operating vehicles of a carrier are all in the same distribution center.

(4) The models of distribution vehicles are the same; that is, the capacity and cost per unit are the same.

(5) The goods are compatible with each other; that is, they can be transported in the same vehicle.

According to graph theory, the problem can be defined as a directed graph $G=(V, A)$. The parameters and symbols are described in Table 1.

According to the above description, the mathematical model can be constructed:

$$
\min \sum_{h \in H} \sum_{i \in V} \sum_{j \in V} x_{i j}^{h} d_{i j} c^{u}+\sum_{h \in H} \sum_{i \in H} \sum_{j \in H} f_{i j}^{h} d_{i j} c^{r}+F \sum_{h \in H} \sum_{j \in C} x_{h j}^{h} \text {, }
$$

s.t.

$$
\begin{aligned}
\sum_{h \in H} \sum_{j \in V} x_{i j}^{h} & =1, \quad \forall i \in C, \\
\sum_{j \in V} x_{i j}^{h} & =\sum_{j \in V} x_{j i}^{h}, \quad \forall i \in V, h \in H, \\
\sum_{i \in V} x_{i j}^{h} & =0, \quad \forall h \in H, j \in H, j \neq h, \\
\sum_{j \in V} f_{h j}^{h} & =Q_{h}, \quad \forall h \in H, \\
\sum_{j \in V} f_{j i}^{h} & -\sum_{j \in V} f_{i j}^{h}=q_{i}^{h}, \quad \forall i \in C, h \in H, \\
\sum_{j \in V} f_{i j}^{h} & =\sum_{j \in V} f_{j i}^{h}, \quad \forall i \in H, h \in H, i \neq h, \\
\sum_{h \in H} f_{i j}^{h} & \leq M u_{i j}, \quad \forall i \in H, j \in H, \\
\sum_{h \in H} f_{i j}^{h} \leq Q & \sum_{h \in H} x_{i j}^{h}, \quad \forall i \in H, j \in C,
\end{aligned}
$$


TABle 1: Notations for model.

\begin{tabular}{lc}
\hline Symbol & Description \\
\hline Sets & Set of customer points \\
$H$ & Set of DC points \\
$V$ & Set of all points \\
Parameters & Distance from $i$ to $j$ \\
$d_{i j}$ & Travel time from $i$ to $j$ \\
$t_{i j}$ & Unit distance cost of distribution vehicles \\
$c^{u}$ & Transfer cost per unit distance per unit volume of goods \\
$c^{r}$ & Fixed cost of vehicles \\
$F$ & Maximum vehicle load \\
$Q$ & Initial cargo volume of distribution center $h$ \\
$Q_{h}$ & Demand for goods of customer $i$ in distribution center $h$ \\
$q_{i}$ & indicator variable, indicates whether the distribution center is directly connected \\
$u_{i j}$ & Earliest service time of customer $i$ \\
$l_{i}$ & Latest service time of customer $i$ \\
$e_{i}$ & Service time of customer $i$ \\
$s_{i}$ & \\
\hline Decision variables & \\
$f_{i j}^{h}$ & Value \\
$x_{i j}^{h}$ & Time that vehicle starts to serve customer $i$ \\
$\tau_{i}$ &
\end{tabular}

$$
\begin{gathered}
\sum_{h \in H} f_{i j}^{h} \leq Q \sum_{h \in H} x_{i j}^{h}, \quad \forall i \in C, j \in H, \\
\sum_{h \in H} f_{i j}^{h} \leq Q \sum_{h \in H} x_{i j}^{h}, \quad \forall i \in C, j \in C, \\
\tau_{i}+s_{i}+t_{i j}-\sum_{h \in H} M\left(1-x_{i j}^{h}\right) \leq \tau_{j}, \quad \forall i \in C, j \in C, \\
e_{i} \leq \tau_{i} \leq l_{i}, \quad \forall i \in C, \\
f_{i j}^{h} \geq 0, \quad \forall i, j \in V, h \in H, \\
x_{i j}^{h} \in\{0,1\}, \quad \forall i, j \in V, h \in H .
\end{gathered}
$$

The objective function (1) is to minimize the total cost, which consists of the transportation cost of distribution vehicles, the transfer cost of outer ring. and the fixed vehicle cost.

Constraint (2) means that each customer can only be served by one vehicle; constraint (3) is vehicle flow balance constraint.

Constraint (4) stipulates that each vehicle must return to the original distribution center; constraint (5) is the outflow constraint of goods from the distribution center.

Constraint (6) means to meet the needs of each customer; constraint (7) ensures the flow balance of distribution center.

Constraint (8) means that goods exchanged between distribution centers can only be carried out through outer ring, where $M$ is a large enough real number.

Constraints (9)-(11) indicate that the capacity constraints of distribution vehicles are met.
Constraints (12) and (13) indicate that the delivery vehicle must meet the time window constraint; constraint (14) indicates that the freight flow on the road network must be nonnegative.

Constraint (15) is a $0-1$ variable constraint.

This problem is a variant of vehicle routing problem, so it is also an NP-hard problem, which is solved by heuristic algorithm. In many heuristic algorithms, in view of the successful application of variable neighborhood search algorithm in VRP, this method is adopted to design the algorithm of this problem.

In addition, what is given here is a general problem description and mathematical model, and there is no specific scenario for a certain kind of cargo transportation, but the model can still be well extended to some application scenarios in urban distribution. For an example of a public distribution center, it represents that the carrier starts from a starting point and its order exchange behavior occurs in the same point. Corresponding to the model, set the distribution center coordinates to the same point, and set the transfer cost to 0. For another example, in supermarket distribution, there may be public customers (as shown in Figure 1), which means that supermarkets have service requirements for different carriers. Corresponding to the model, the coordinates of customer points can be set to the same point. However, if there are new requirements on the constraints, such as the return demand of express delivery, the model needs to be modified accordingly.

\section{Algorithm Principle}

Variable neighborhood search (VNS) is a simple but powerful metaheuristic algorithm. It was first proposed by Mladenovic and Hansen [16]. Its core idea is to 


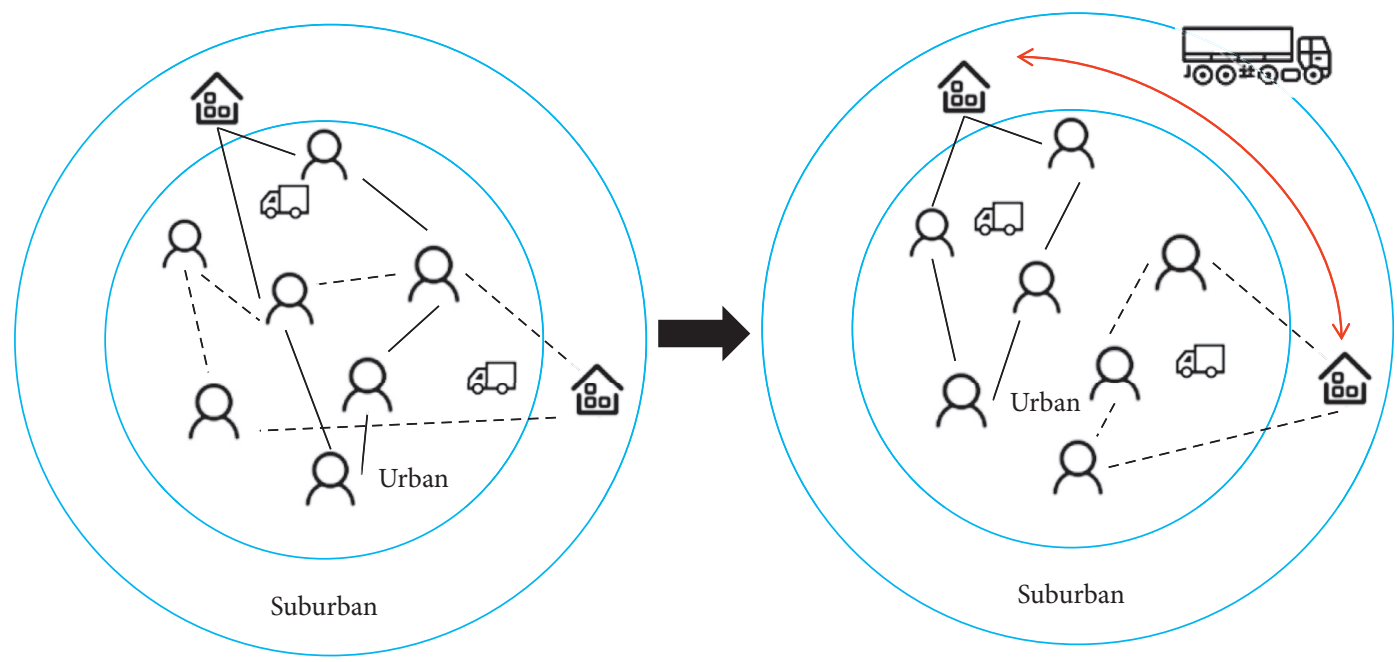

FIgURE 1: Schematic diagram of urban circular distribution network.

systematically and regularly change neighborhood in the process of local search to enhance the search ability of the algorithm. The key lies in the design of neighborhood structure and the strategy of local search. So far, variable neighborhood search has achieved great success in solving integer programming, mixed-integer programming, and nonlinear programming. Variable neighborhood search can be applied to many fields, and it is increasing rapidly, such as location problem, scheduling problem, vehicle routing optimization problem, network communication design, clustering analysis, artificial intelligence, reliability, geometry problem, and overall planning problem.

Variable neighborhood search is a met heuristic algorithm, which changes neighborhood constantly in the process of local optimization and jumping out of local optimization. The so-called neighborhood is simply the set of other points near a given point. In distance space, neighborhood is generally defined as a circle with a given point as its center, while in combinatorial optimization problems, neighborhood is generally defined as the set of nodes in the problem domain obtained by transforming each node in a given problem domain with a given transformation rule.

Variable neighborhood search is based on the following theorems:

(1) The local optimal value in one neighborhood is not necessarily the local optimal value in another neighborhood.

(2) The global optimal value is the local optimal value in all possible neighborhoods.

(3) For many problems, the local minima in different neighborhood are close.

The basic process of variable neighborhood search is as follows: starting from an initial solution, the neighborhood solution is generated according to the current neighborhood structure jitters, and a new solution is obtained by local search of the neighborhood solution; if the new solution is better than the historical optimal solution, the new solution is used as the current solution to continue searching in the neighborhood structure; otherwise, the next neighborhood is turned, and the above process is repeated until it reaches the maximum number of iterations or the generations of the maximum optimal solution that are not updated or the set time limit.

The framework of the typical variable neighborhood search algorithm is shown in Algorithm 1.

In short, VND means that when a better solution cannot be found in the local neighborhood, it will jump to the next neighborhood to continue the search; when a better solution is found in the local neighborhood, it will jump back to the first neighborhood and start the search again. In addition, the search strategies of local search algorithms usually include first improvement and best improvement. The former means that, in the process of solving, if the new solution is better than the current solution, the current solution is updated with the new solution, and the iterative search is carried out again until the current solution cannot be optimized, and it is taken as the final optimal solution. The latter means that, in the process of solving, all the neighborhood solutions of the current solution are traversed, and the solution with the largest improvement range is the final optimal solution. In general, the former is of high quality and the latter is short in solving time.

\section{Algorithm Design}

5.1. Representation of Solutions. Compared with the traditional vehicle routing problem, this paper also handles the problem that vehicles start from different distribution centers, which is similar to the vehicle routing problem with multiple depots. The following code is defined to represent the structure of the solution: $\pi_{i}=\left(u_{h}, c_{1}, c_{2}, \ldots, c_{n}, u_{h}\right)$ means a vehicle $i$ starts from DC $h$ and visits customers $c_{1}, c_{2}, \ldots, c_{n}$ in sequence and then returns to $h$. All customer numbers in all codes appear once and only once (Figure 2).

In addition, when $u_{h}$ is set as the same distribution center, it means vehicles in $u_{h}$ do not transfer. This algorithm 


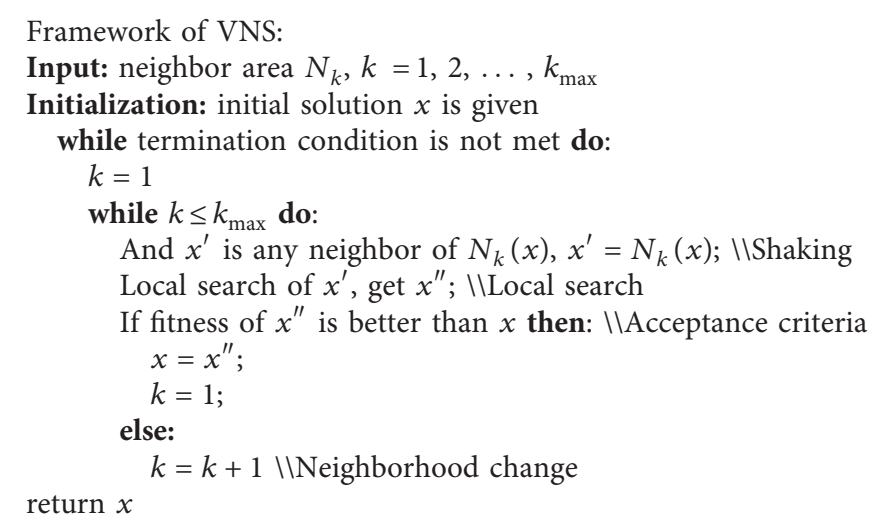

Algorithm 1: Framework of VNS.

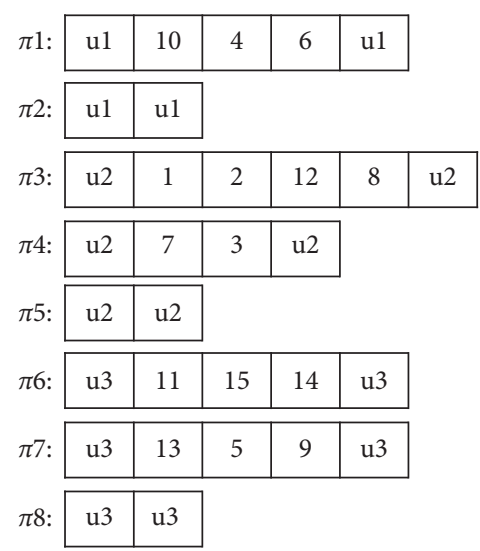

FIgURE 2: Examples of solutions representation in Co-VRP-URLN.

can be directly applied to the ordinary vehicle routing problem with time windows. In numerical experiments, the effectiveness of the algorithm will be tested.

5.2. Generation of Initial Solution and Termination Conditions. The quality of the initial solution affects the efficiency of the algorithm. In this paper, the result of each carrier's distribution before joint distribution is taken as the initial solution. This initial solution provides a good upper bound, which can accelerate the convergence of the algorithm. When solving the vehicle routing problem before joint distribution, the initial solution is simply set as that each customer is served by a single vehicle.

The termination condition is set to stop searching when the optimal solution has not been updated after a given number of iterations or when the maximum CPU time is reached.

5.3. Shaking. The shaking process first selects a predefined neighborhood structure in a certain way and then changes the current solution according to the neighborhood structure to generate a new solution to jump out of the local optimum. The Co-VRP-URLN involves multiple paths.
According to its characteristics, the shaking process is defined as the insertion or cross-exchange of two subpaths.

Insertion: Two paths, $\pi_{i}$ and $\pi_{j}$, are randomly selected from the current solution, and a subpath of length $L_{r}$ from $\pi_{i}$ is selected and then inserted into $\pi_{j}$ (Figure 3 ). Cross-exchange: Two paths, $\pi_{i}$ and $\pi_{j}$, are randomly selected from the current solution, and a subpath of length $L_{r}$ from $\pi_{i}$ and a subpath of length $L_{j}$ from $\pi_{j}$ are selected and then exchanged (Figure 4 ).

It is worth noting that insertion can be seen as a special exchange; one of the selected subpaths has a length of 0 , so they can be combined in programming. In addition, since the existence of infeasible solutions is allowed, it is not necessary to judge whether the operation is feasible. They are classified into different neighborhoods according to different operations, different length of route selection, and different sources of routes (which can be divided into the same distribution center and different distribution center) (Figure 4).

5.4. Local Search. Local search is defined as two or three paths involved in shaking to optimize their respective paths. Local search is the most time-consuming part of the whole VNS algorithm and largely determines the final solution quality. Therefore, both time and quality should be considered in the design of local search algorithm. In this paper, two local search methods are proposed. The first is to use VND for local search, and the second is to solve the problem in accurate method.

5.4.1. VND. For the path optimization, the classical heuristic optimization operator $k$-opt is used as the neighborhood structure, which represents the exchange of $k$ edges of an itinerant path. A large number of numerical experiments show that the local optimal solution of 3-opt is better than 2opt and 4-opt is slightly better than 3-opt, but it takes too long. In addition, or-opt operator is a subset of 3-opt operator and can obtain similar quality solutions in a shorter time. Therefore, 2-opt operator and or-opt operator are 

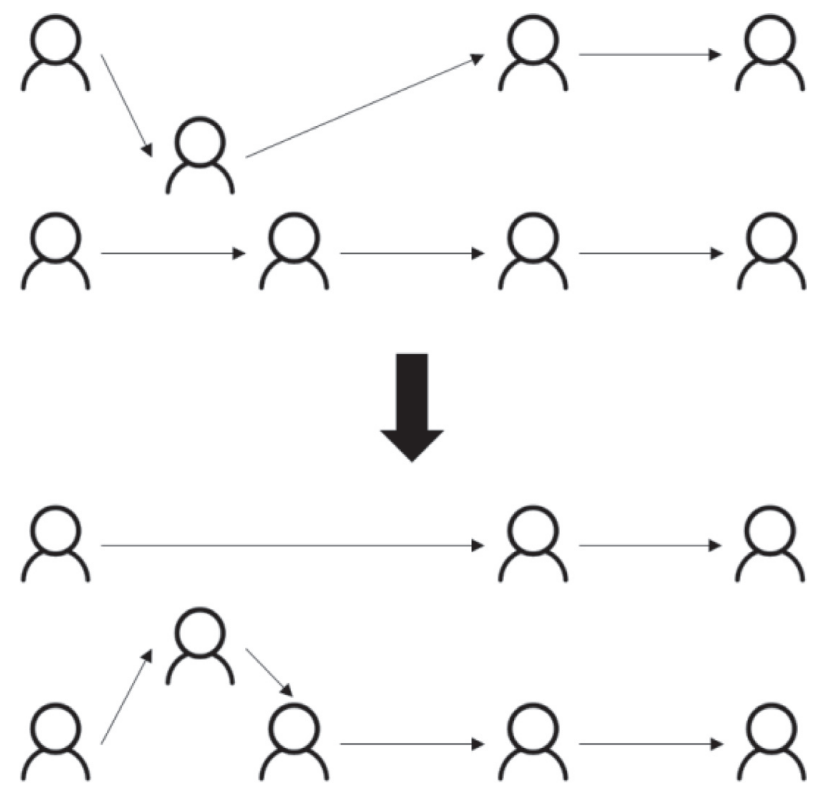

Figure 3: An example of insertion.
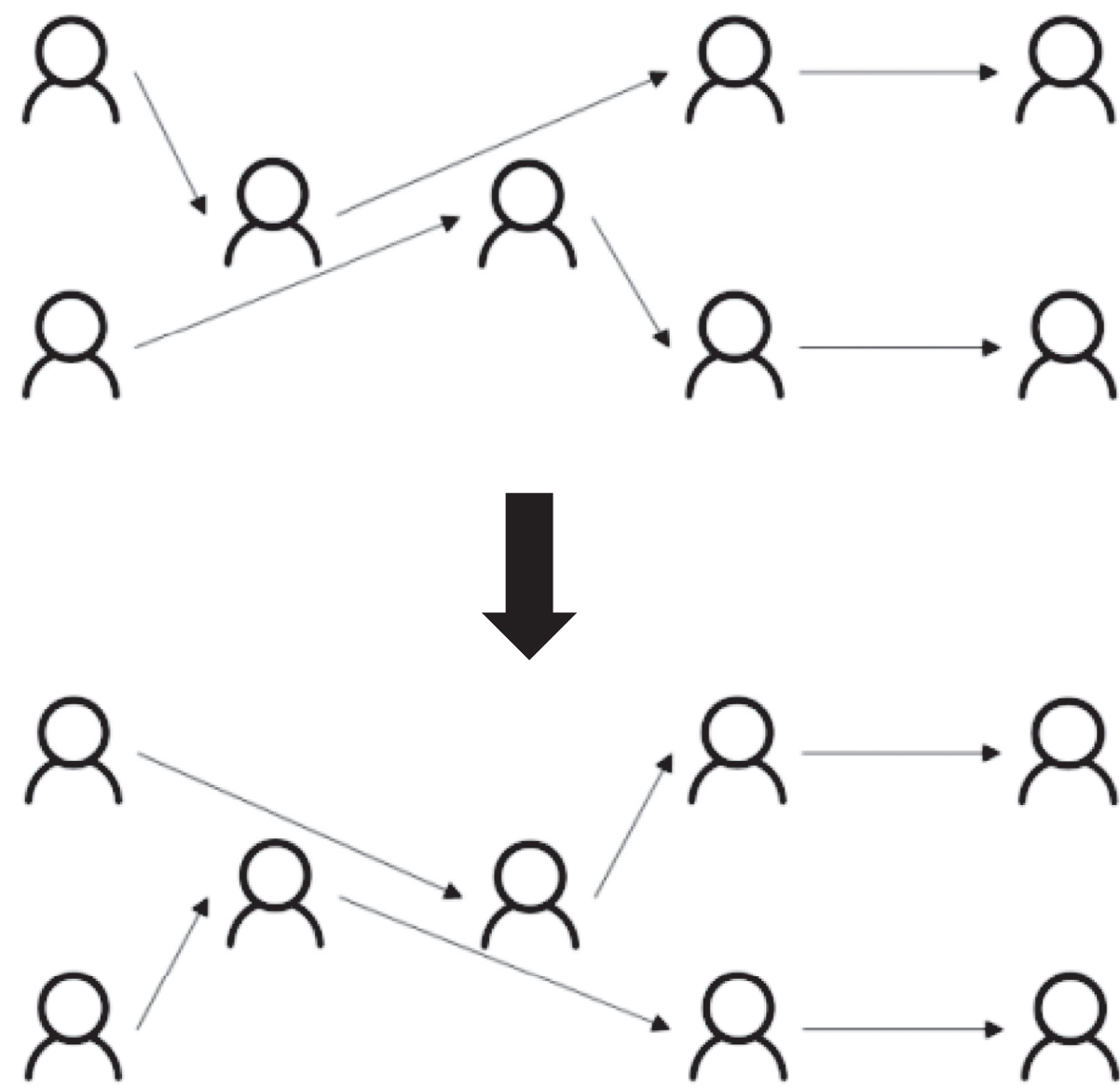

FIgURE 4: An example of cross-exchange.

selected as the neighborhood structure of local search. After the preexperiment, the first improvement strategy is adopted to speed up the search.
5.4.2. Accurate Solution. Local path optimization is also a traveling salesman problem with time window (TSPTW). The model is described as follows: 
$\min \sum_{i \in V} \sum_{j \in V} c_{i j} x_{i j}+\alpha \max \left\{0, \sum_{i \in V} q_{i}-Q\right\}+\beta \sum_{i \in C} \max \left\{0, \tau_{i}-l_{i}\right\}$,

s.t.

$$
\begin{gathered}
\sum_{i \in V} x_{i j}=1, \quad \forall j \in V, \\
\sum_{j \in V} x_{i j}=1, \quad \forall i \in V, \\
\tau_{i}+s_{i}+t_{i j}-M\left(1-x_{i j}\right) \leq \tau_{j}, \quad \forall i, j \in C, \\
\tau_{i} \geq e_{i}, \quad \forall i \in C, \\
x_{i j} \in\{0,1\}, \quad \forall i, j \in V, \\
\tau_{i} \geq 0, \quad \forall i \in V .
\end{gathered}
$$

Although the TSPTW is also an NP-hard problem, considering the capacity constraint when shaking, a path does not contain too many customers. Therefore, the solver can be used to obtain the optimal solution of the above problem in a short time. It should be noted that when the optimal solution is obtained, the coding should be arranged according to the format of the optimal solution.

$$
\begin{aligned}
& u=\max \left\{0, \sum_{i \in V} q_{i}-Q\right\}, \\
& v_{i}=\max \left\{0, \tau_{i}-l_{i}\right\} .
\end{aligned}
$$

Then the objective function becomes

$$
\min \sum_{i \in V} \sum_{j \in V} c_{i j} x_{i j}+\alpha u+\beta \sum_{i \in C} v_{i},
$$

s.t.

$$
\begin{aligned}
& u \geq 0, \\
& u \geq \sum_{i \in V} q_{i}-Q, \\
& v_{i} \geq 0, \quad \forall i \in C, \\
& v_{i} \geq \tau_{i}-l_{i}, \quad \forall i \in C .
\end{aligned}
$$

It can be seen that the model composed of (25), (17)-(22), and (26)-(29) is equivalent to the original model and is a linear-integer programming. In the implementation of local search in this paper, when the number of points on the path is small, the exact algorithm is used, and when the number is large, VND is used.

5.5. Acceptance Criteria of Solutions. The acceptance criteria determine which solution is accepted for the next iteration. In this algorithm, the Metropolis criterion in simulated annealing algorithm is used to select the solution to enter the next iteration. The criterion can accept the worse solution with a certain probability and avoid falling into local optimum prematurely. The criterion is shown in equation (30), where $\gamma$ is the random number in the interval $[0,1]$; the initial temperature $T$ is $T_{0}$, which is updated every other generation according to $T_{n+1}=\theta T_{n} ; \theta$ is the settable cooling coefficient.

$$
\mathrm{SA}\left(x^{\prime}, x\right)=\left\{\begin{array}{l}
x^{\prime}, \gamma \geq \exp \left(\frac{f\left(x^{\prime}\right)-f(x)}{T}\right), \\
x, \gamma<\exp \left(\frac{f\left(x^{\prime}\right)-f(x)}{T}\right) .
\end{array}\right.
$$

\section{Numerical Experiment}

6.1. Example Construction. Since there is no specific data for this problem, it is necessary to construct an example. Based on the standard example of vehicle routing problem with time windows (VRPTW) proposed by Solomon in 1987, the corresponding improvement is made, and a series of test data sets are obtained, which are used to test the effectiveness of the algorithm, and the benefits of joint allocation are explained. The example can be downloaded from Solomon's official website (source: http://web.cba.neu.edu/\%7Emsolomon/problems.htm).

The geographical location and time window of customer points are given in Solomon's standard data set. The geographical location is divided into three categories: R, C, and $\mathrm{RC}$, where $\mathrm{R}$ represents random and $\mathrm{C}$ represents cluster. The three categories correspond to random distribution, aggregation distribution, and mixed distribution of customer points, as shown in Figure 5. Each class gives three different size data sets of 25,50 , and 100 customer points. Considering that the effect of joint distribution is not obvious if there are few customers, and too many customers will affect the calculation speed, this section uses the data set of 50 customer points with moderate scale. Furthermore, considering that urban distribution is mainly random distribution and generally has tight time window, this paper selects "r102-50" in class R as the basic example and constructs it on the basis of customer distribution and time window.

Each example in Solomon's data set only gives the location of one distribution center; according to the demand of this problem, we need to design several reasonable distribution center locations. Therefore, a simple method to determine the location of distribution center is proposed as follows: Firstly, the centroid of all customer points is calculated, and the distance from the farthest customer point to the point is taken as the radius to make a circle. Finally, some points on the circle are selected as the geographical location of the distribution center, and customers are randomly assigned to one of the carriers with equal probability. In addition, the distance between customers and the distribution center is calculated by Euclidean distance, and the 

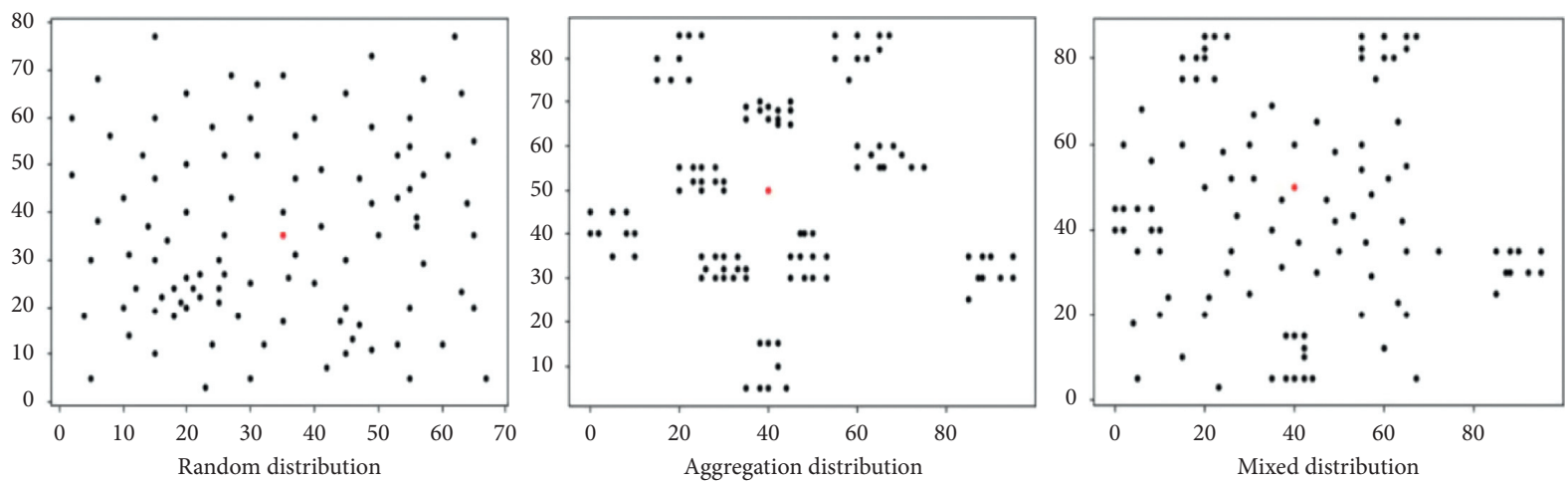

FIgURE 5: Three kinds of examples of Solomon's standard calculation.

distance between distribution centers is calculated according to the arc length of the circle.

Taking three carriers ( $\mathrm{a}, \mathrm{b}$ and $\mathrm{c}$ ) as examples, this paper designs three ways to select distribution centers, which represent the distribution forms of three distribution centers: far, near-far, and near, which are called $X, Y$, and $Z$ respectively. Specifically, in class $X,(\mathrm{a}, \mathrm{b}, \mathrm{c})$ is fixed at $0^{\circ}$, $120^{\circ}$, and $240^{\circ}$, respectively; in class $Y,(\mathrm{a}, \mathrm{b}, \mathrm{c})$ is fixed at $0^{\circ}$, $150^{\circ}$, and $210^{\circ}$; finally, in class $Z,(\mathrm{a}, \mathrm{b}, \mathrm{c})$ is fixed at $0^{\circ}, 60^{\circ}$, and $300^{\circ}$, respectively. The distribution center of the three kinds of examples is shown in Figure 6. In order to conduct sufficient numerical experiments, 10 different assignments are carried out in random assignment of customer points, with a total of 30 examples. Use " $M-N$ " to represent an example with category $M$ and number $N$. For example, " $X-1$ " represents an example of class $X$ with no. 1 .

6.2. Parameter Setting. Parameter setting includes model input and algorithm superparameter setting. The input of the model is designed reasonably according to the actual situation, and the superparameters of the algorithm are determined by preexperiment. The parameter settings used in this section are shown in Table 2 .

6.3. Experimental Results of Standard Data Set. In order to test the effectiveness of the algorithm, experiments are carried out on the standard VRPTW problem and compared with best known solutions (BKS). Note that the vehicle cost is not included in the standard VRPTW problem, so it needs to be modified in the model input.

Specifically, firstly, in order to test the stability of the algorithm and reduce the experimental error, 10 numerical experiments are carried out on 12 R-class examples of 50 customer points in Solomon's standard data set. The worst results, best results, and average results are recorded. The average usage of vehicles is also given, which is expressed by BEST, AVG, WORST, and KAVG, respectively. Secondly, three results are compared with the known optimal solution $\mathrm{BKS}$. The calculation uses the general comparison method: (SOL-BKS)/SOL $\times 100 \%$, where SOL is the result obtained by this algorithm, and the comparison results are represented by $\mathrm{Gap}_{\mathrm{K}}$, Gap $\mathrm{G}_{\mathrm{BEST}}$, Gap $\mathrm{AVG}$, and Gapworst, respectively. Table 3 shows the experimental results.
It can be seen that, in 12 examples, the percentage error of the average result obtained by this algorithm is $2 \%-6 \%$; in the optimal performance of the algorithm, the optimal solution is obtained for R101, R104, and R108 problems, and the maximum percentage error is $3.54 \%$ (R107); the percentage error of the worst performance is $5 \%-9 \%$, which is still within the acceptable range.

In general, the comparison with the known optimal solution shows that the algorithm can approach the optimal solution in a better way and even achieve the optimal solution in some examples, which shows the effectiveness of the algorithm, and, from the results of average performance and worst performance, the algorithm also has good stability. Therefore, the result of applying the algorithm to this model should be more reliable.

6.4. Evaluation of Joint Distribution. Next, the algorithm is applied to this problem, and the results before and after joint distribution are compared in the constructed example. For the stability of the results, each example is still calculated 10 times, and the average value is taken as the final result. The experimental results include the total distribution cost (COST), the required vehicle (K), and path length (DIS) of the carrier before joint distribution, as well as the total distribution cost (COST), the required vehicle $(\mathrm{K})$, path length (DIS), and transfer cost (TRANS) after joint distribution, and the percentage gap (GAP) between them is given. The results of $X, Y$, and $Z$ cases are shown in Tables 4-6, respectively.

6.4.1. Cost Analysis before and after Joint Distribution. The results in the table show that the joint distribution can bring about quite ideal cost reduction. The cost reductions of $X, Y$, and $Z$ cases are $35.16 \%, 32.61 \%$, and $29.95 \%$, respectively. In terms of different cost reduction percentages, the vehicle costs of the three types of calculation cases were reduced by $28.86 \%, 29.15 \%$, and $18.75 \%$, respectively, and the transportation costs were reduced by $48.24 \%, 42.87 \%$, and $26.34 \%$, which shows that, through joint distribution, the use of vehicles and transportation distance of the whole alliance have been further optimized, which can not only produce economic benefits but also bring social benefits by reducing distribution vehicles and distribution distance. 

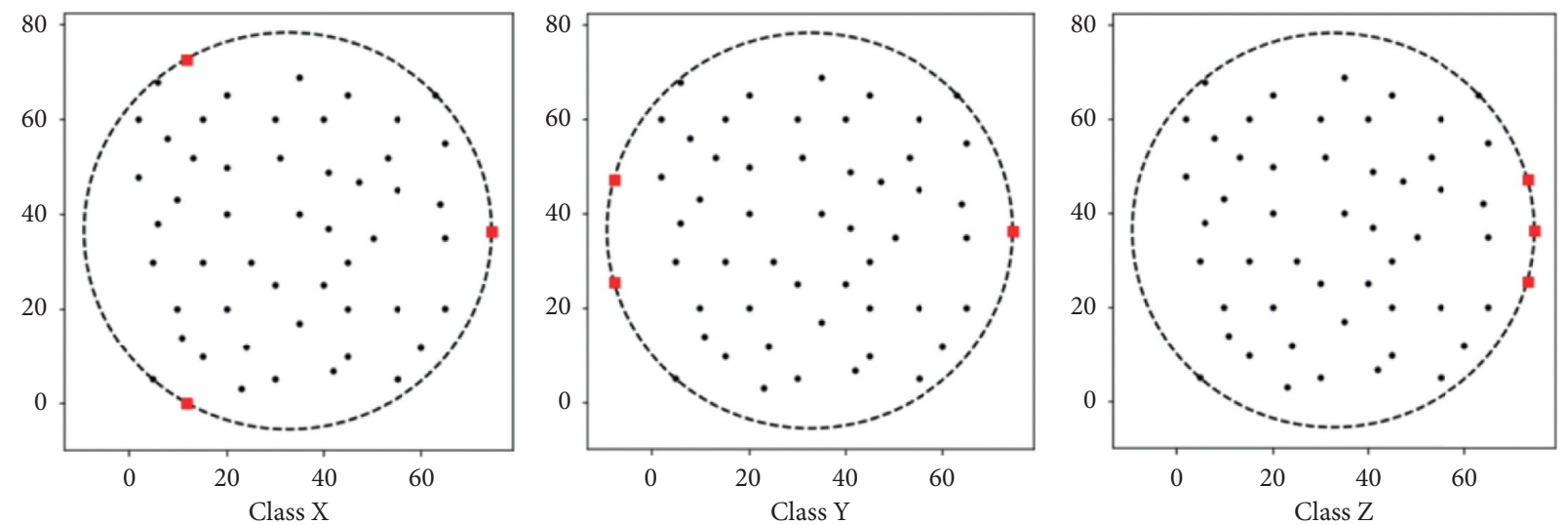

FIgure 6: Three classes of distribution of DC.

Table 2: Parameter setting.

\begin{tabular}{lcc}
\hline Parameter & Description & Value \\
\hline$F$ & Fixed cost of vehicle & 150 \\
$c^{u}$ & Unit distance cost of distribution vehicles & 1 \\
$c^{r}$ & Transfer cost per unit distance per unit volume of goods & 0.01 \\
$\alpha$ & Penalty coefficient of capacity constraint & 100 \\
$\beta$ & Penalty coefficient of time window constraint & 100 \\
Flag & The number of iterations without updating the maximum optimal solution & 5000 \\
$T_{0}$ & Initial temperature & 10000 \\
$\theta$ & Cooling coefficient & 0.9999 \\
\hline
\end{tabular}

TABLE 3: VRPTW experimental results of standard data set.

\begin{tabular}{|c|c|c|c|c|c|c|c|c|c|c|}
\hline \multirow{2}{*}{ Example } & \multicolumn{3}{|c|}{ BKS } & \multicolumn{3}{|c|}{ SOL } & \multicolumn{4}{|c|}{ Gap (\%) } \\
\hline & $\mathrm{K}$ & BKS & $\mathrm{K}_{\mathrm{AVG}}$ & BEST & AVG & WORST & $\operatorname{Gap}_{\mathrm{K}}(\%)$ & $\operatorname{Gap}_{\text {BEST }}(\%)$ & $\operatorname{Gap}_{\mathrm{AVG}}(\%)$ & Gap $_{\text {WORST }}(\%)$ \\
\hline R101 & 12 & 1044.0 & 12.6 & 1044.0 & 1066.9 & 1106.0 & 4.76 & 0.00 & 2.15 & 5.61 \\
\hline $\mathrm{R} 102$ & 11 & 909.0 & 11.7 & 927.3 & 941.9 & 966.1 & 5.98 & 1.97 & 3.49 & 5.91 \\
\hline R103 & 9 & 772.9 & 9.3 & 788.0 & 810.6 & 840.0 & 3.23 & 1.92 & 4.65 & 7.99 \\
\hline R104 & 6 & 625.4 & 6.4 & 625.4 & 643.9 & 662.3 & 6.25 & 0.00 & 2.87 & 5.57 \\
\hline R105 & 9 & 899.3 & 9.5 & 910.4 & 940.6 & 959.9 & 5.26 & 1.22 & 4.39 & 6.31 \\
\hline R106 & 8 & 793.0 & 8.7 & 793.0 & 832.1 & 854.8 & 8.05 & 0.00 & 4.70 & 7.23 \\
\hline R107 & 7 & 711.1 & 7.3 & 737.2 & 733.3 & 750.2 & 4.11 & 3.54 & 3.03 & 5.21 \\
\hline R108 & 6 & 617.7 & 6.3 & 617.7 & 639.9 & 659.4 & 4.76 & 0.00 & 3.46 & 6.32 \\
\hline R109 & 8 & 786.8 & 8.7 & 795.0 & 816.3 & 843.5 & 8.05 & 1.03 & 3.62 & 6.72 \\
\hline $\mathrm{R} 110$ & 7 & 697.0 & 7.6 & 712.1 & 735.9 & 759.7 & 7.89 & 2.12 & 5.29 & 8.25 \\
\hline R111 & 7 & 707.2 & 7.7 & 715.7 & 754.7 & 783.1 & 9.09 & 1.19 & 6.30 & 9.69 \\
\hline $\mathrm{R} 112$ & 6 & 630.2 & 6.5 & 646.8 & 668.2 & 682.7 & 7.69 & 2.57 & 5.69 & 7.69 \\
\hline
\end{tabular}

Table 4: Result of $X$ class example.

\begin{tabular}{|c|c|c|c|c|c|c|c|c|c|c|}
\hline \multirow{2}{*}{ Example } & \multicolumn{3}{|c|}{ Before joint distribution } & \multicolumn{3}{|c|}{ After joint distribution } & \multicolumn{4}{|c|}{ Gap (\%) } \\
\hline & COsT & $\mathrm{K}$ & DIS & COST & $\mathrm{K}$ & DIS & TRANS & COST & $\mathrm{K}$ & DIS \\
\hline$X-1$ & 4797.1 & 18.0 & 2097.1 & 3216.5 & 12.7 & 1214.6 & 101.9 & $32.95 \%$ & $29.63 \%$ & $42.08 \%$ \\
\hline$X-2$ & 4857.8 & 18.7 & 2057.8 & 3149.5 & 13.0 & 1095.6 & 103.9 & $35.17 \%$ & $30.36 \%$ & $46.76 \%$ \\
\hline$X-3$ & 5033.6 & 19.3 & 2133.6 & 3232.5 & 13.3 & 1120.1 & 112.4 & $35.78 \%$ & $31.03 \%$ & $47.50 \%$ \\
\hline$X-4$ & 4897.5 & 18.7 & 2097.5 & 3140.9 & 13.0 & 1080.1 & 110.8 & $35.87 \%$ & $30.36 \%$ & $48.50 \%$ \\
\hline$X-5$ & 5024.9 & 18.3 & 2274.9 & 3149.8 & 13.0 & 1077.2 & 122.6 & $37.32 \%$ & $29.09 \%$ & $52.65 \%$ \\
\hline$X-6$ & 4639.9 & 17.7 & 1989.9 & 3132.2 & 13.0 & 1099.2 & 83.0 & $32.49 \%$ & $26.42 \%$ & $44.76 \%$ \\
\hline$X-7$ & 4789.3 & 18.0 & 2089.3 & 3251.8 & 13.7 & 1087.9 & 113.9 & $32.10 \%$ & $24.07 \%$ & $47.93 \%$ \\
\hline$X-8$ & 4842.7 & 17.7 & 2192.7 & 3040.9 & 12.3 & 1116.1 & 74.8 & $37.21 \%$ & $30.19 \%$ & $49.10 \%$ \\
\hline$X-9$ & 5236.2 & 19.7 & 2286.2 & 3052.6 & 12.7 & 1032.1 & 120.5 & $41.70 \%$ & $35.59 \%$ & $54.86 \%$ \\
\hline$X-10$ & 4740.4 & 17.7 & 2090.4 & 3311.9 & 14.0 & 1106.6 & 105.3 & $30.14 \%$ & $20.75 \%$ & $47.06 \%$ \\
\hline Avg & 4885.9 & 18.4 & 2130.9 & 3167.9 & 13.1 & 1102.9 & 104.9 & $35.16 \%$ & $28.86 \%$ & $48.24 \%$ \\
\hline
\end{tabular}


TABLe 5: Result of $Y$ class example.

\begin{tabular}{|c|c|c|c|c|c|c|c|c|c|c|}
\hline \multirow{2}{*}{ Example } & \multicolumn{3}{|c|}{ Before joint distribution } & \multicolumn{3}{|c|}{ After joint distribution } & \multicolumn{4}{|c|}{ Gap (\%) } \\
\hline & Cost & K & DIS & COsT & K & DIS & COST & $\mathrm{K}$ & DIS & COsT \\
\hline$Y-1$ & 5185.6 & 19.3 & 2285.6 & 3344.9 & 13.3 & 1188.2 & 156.7 & $35.50 \%$ & $31.03 \%$ & $48.02 \%$ \\
\hline$Y-2$ & 4455.1 & 16.7 & 1955.1 & 3231.5 & 12.7 & 1255.8 & 75.7 & $27.47 \%$ & $24.00 \%$ & $35.77 \%$ \\
\hline$Y-3$ & 5027.8 & 18.7 & 2227.8 & 3235.2 & 12.7 & 1182.2 & 153.0 & $35.65 \%$ & $32.14 \%$ & $46.93 \%$ \\
\hline$Y-4$ & 4727.8 & 18.0 & 2027.8 & 3230.3 & 12.7 & 1205.3 & 125.0 & $31.67 \%$ & $29.63 \%$ & $40.56 \%$ \\
\hline$Y-5$ & 4452.5 & 16.7 & 1952.5 & 3251.6 & 12.7 & 1235.2 & 116.4 & $26.97 \%$ & $24.00 \%$ & $36.74 \%$ \\
\hline$Y-6$ & 4965.9 & 18.7 & 2165.9 & 3292.0 & 13.0 & 1212.5 & 129.5 & $33.71 \%$ & $30.36 \%$ & $44.02 \%$ \\
\hline$Y-7$ & 4368.7 & 17.0 & 1818.7 & 3233.3 & 13.0 & 1200.4 & 82.9 & $25.99 \%$ & $23.53 \%$ & $33.99 \%$ \\
\hline$Y-8$ & 4909.5 & 17.3 & 2309.5 & 3272.0 & 12.7 & 1212.8 & 159.2 & $33.35 \%$ & $26.92 \%$ & $47.49 \%$ \\
\hline$Y-9$ & 4983.1 & 18.3 & 2233.1 & 3165.9 & 12.3 & 1188.2 & 127.8 & $36.47 \%$ & $32.73 \%$ & $46.79 \%$ \\
\hline$Y-10$ & 5281.5 & 20.0 & 2281.5 & 3330.3 & 13.0 & 1263.8 & 116.4 & $36.95 \%$ & $35.00 \%$ & $44.61 \%$ \\
\hline Avg & 4835.7 & 18.1 & 2125.7 & 3258.7 & 12.8 & 1214.4 & 124.3 & $32.61 \%$ & $29.15 \%$ & $42.87 \%$ \\
\hline
\end{tabular}

TABLe 6: Result of $Z$ class example.

\begin{tabular}{|c|c|c|c|c|c|c|c|c|c|c|}
\hline \multirow{2}{*}{ Example } & \multicolumn{3}{|c|}{ Before joint distribution } & \multicolumn{3}{|c|}{ After joint distribution } & \multicolumn{4}{|c|}{ Gap (\%) } \\
\hline & COST & $\mathrm{K}$ & DIS & COsT & $\mathrm{K}$ & DIS & TRANS & COST & $\mathrm{K}$ & DIS \\
\hline$Z-1$ & 5020.2 & 19.0 & 2170.2 & 3511.6 & 12.7 & 1517.0 & 94.6 & $30.05 \%$ & $33.33 \%$ & $30.10 \%$ \\
\hline$Z-2$ & 5090.0 & 19.0 & 2240.0 & 3776.5 & 14.0 & 1557.3 & 119.2 & $25.80 \%$ & $26.32 \%$ & $30.48 \%$ \\
\hline$Z-3$ & 4781.4 & 18.0 & 2081.4 & 3612.9 & 13.3 & 1533.7 & 79.2 & $24.44 \%$ & $25.93 \%$ & $26.31 \%$ \\
\hline$Z-4$ & 4730.8 & 17.3 & 2130.8 & 3732.2 & 13.7 & 1577.1 & 105.1 & $21.11 \%$ & $21.15 \%$ & $25.98 \%$ \\
\hline$Z-5$ & 4780.5 & 17.7 & 2130.5 & 3725.1 & 13.3 & 1620.9 & 104.2 & $22.08 \%$ & $24.53 \%$ & $23.92 \%$ \\
\hline$Z-6$ & 5592.7 & 20.7 & 2492.7 & 3770.0 & 13.7 & 1587.8 & 132.3 & $32.59 \%$ & $33.87 \%$ & $36.30 \%$ \\
\hline$Z-7$ & 4850.4 & 18.7 & 2050.4 & 3641.8 & 13.0 & 1598.1 & 93.8 & $24.92 \%$ & $30.36 \%$ & $22.06 \%$ \\
\hline$Z-8$ & 4636.8 & 17.3 & 2036.8 & 3656.8 & 13.3 & 1545.4 & 111.4 & $21.13 \%$ & $23.08 \%$ & $24.12 \%$ \\
\hline$Z-9$ & 4886.7 & 18.0 & 2186.7 & 3599.7 & 13.0 & 1555.7 & 94.0 & $26.34 \%$ & $27.78 \%$ & $28.86 \%$ \\
\hline$Z-10$ & 4498.1 & 16.6 & 2008.1 & 3041.8 & 10.9 & 1324.2 & 82.6 & $32.38 \%$ & $34.34 \%$ & $34.06 \%$ \\
\hline Avg & 4511.8 & 16.0 & 2111.8 & 3611.7 & 13.0 & 1555.6 & 106.1 & $19.95 \%$ & $18.75 \%$ & $26.34 \%$ \\
\hline
\end{tabular}

TABLe 7: Cost analysis before and after joint distribution.

\begin{tabular}{|c|c|c|c|c|c|c|c|c|}
\hline \multirow{2}{*}{ Examples } & \multicolumn{4}{|c|}{ Before joint distribution } & \multicolumn{4}{|c|}{ After joint distribution } \\
\hline & Total cost & Cost of vehicle & Cost of distance & Transfer cost & Total cost & Cost of vehicle & Cost of distance & Transfer cost \\
\hline Avg & 4744.51 & 2621.67 & 2122.84 & 0.00 & 3346.09 & 1943.33 & 1291.00 & 111.76 \\
\hline Prop. & - & $55.26 \%$ & $44.74 \%$ & $0.00 \%$ & - & $58.08 \%$ & $38.58 \%$ & $3.34 \%$ \\
\hline
\end{tabular}

From the perspective of cost proportion, Table 7 shows the average cost and the proportion of each cost source before and after joint distribution. The proportion of each cost source is given in a more intuitive way in Figure 7.

It can be found that the proportion of vehicle cost increased from $55.26 \%$ to $58.08 \%$, while the proportion of distance cost decreased from $44.74 \%$ to $38.58 \%$, indicating that transportation cost benefits more in joint distribution. It can be understood that as the total amount of customer demand remains unchanged, there will be a strong lower bound limit on the number of vehicles saved, and the optimization of transportation distance is relatively more flexible. It can be adjusted by transferring according to the geographical location of distribution center and the location of customer points, so there is more space for optimization. In the following, three kinds of examples are compared horizontally to analyze the influence of distribution center distribution.
6.4.2. Influence Analysis of DC Distribution. The examples of $X, Y$, and $Z$ represent the change of distribution center from decentralization to centralization. Figure 8 shows the relationship between the descent and the example.

It can be found that the most decentralized distribution center (class $X$ ) can bring the biggest cost reduction. However, when the distribution centers are relatively concentrated (class $Z$ ), the profit brought by joint distribution is not as good as that brought by decentralized distribution (class $X$ and class $Y$ ), which is more in line with the expectation at the beginning of this paper, because the decentralized distribution center can alleviate the longdistance transportation behavior in the city to a certain extent. Due to the combination of goods and a certain amount of goods, the proportions of vehicle cost reduction are relatively similar. In addition, it should be pointed out that the performances of class $X$ and class $Y$ are very similar, which means that, in the distribution alliance, it is not 


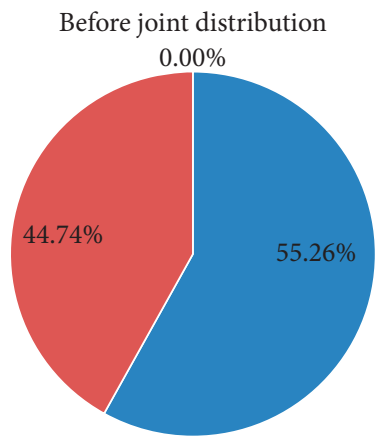

- Cost of vehicle

- Cost of distance

- Transfer cost

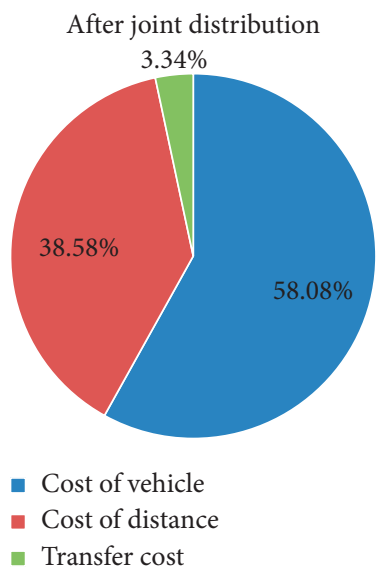

Figure 7: Cost proportion before and after joint distribution.

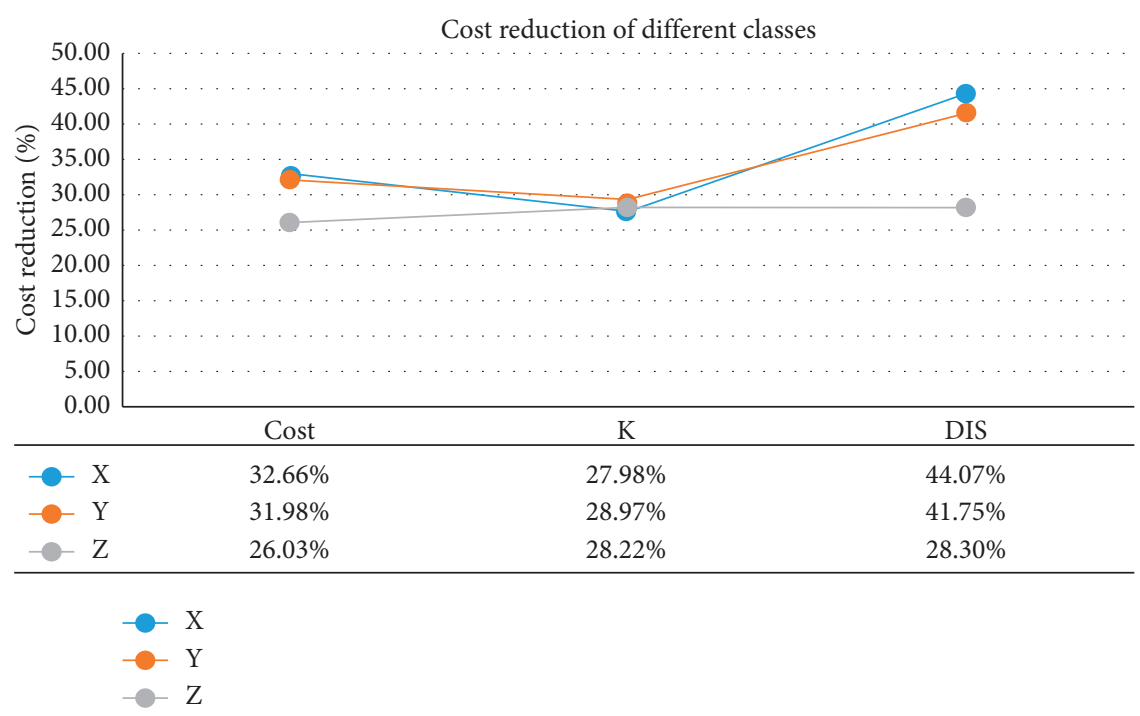

FIgURE 8: Cost reduction of three kinds of examples.

necessary to have a better effect when all the distribution centers are relatively scattered. The transfer cost between similar distribution centers is relatively low, which can be approximately regarded as the same public distribution center, whose orders can be effectively merged and loaded, which is equivalent to two local optimal solutions. If it becomes a global optimal solution, the cost will be reduced accordingly. In other words, different distribution of distribution centers is conducive to the reduction of cost, which may promote the formation of distribution alliance.

Generally speaking, the cost of the whole alliance can be greatly reduced by the joint distribution of the city "ring" network through a small amount of transfer costs, including the reduction of vehicle use and transportation distance, which will produce good economic and social benefits.

\section{Conclusion}

This paper proposes a new research problem and direction based on joint distribution: Co-VRP-URLN. It is a derivative of multidepot vehicle routing problem and multilevel vehicle problem. In this paper, a mixed-integer linear programming is established for this NP-hard problem. According to the framework of variable neighborhood search algorithm (VNS), the solution representation, fitness calculation method, initial solution generation strategy and termination condition, dithering neighborhood structure, and method, two local search strategies and solution acceptance criteria are designed. Through numerical experiments, the effectiveness and stability of the algorithm are verified. At the same time, the change of joint distribution cost under different distribution center distribution conditions is evaluated. The results are basically consistent with the expected results and conform to the characteristics of urban distribution.

Each carrier's vehicle routing planning and order selection are determined by itself rather than collecting all information through a "decision center" for unified planning. Future research will further explore distributed joint distribution, that is, cooperation under the premise of incomplete information sharing. 


\section{Data Availability}

The data are available at http://web.cba.neu.edu/ $\sim$ msolomon/problems.htm.

\section{Conflicts of Interest}

The authors declare that they have no conflicts of interest.

\section{References}

[1] R. Liu, Z. Jiang, and R. Y. K. Fung, "Two-phase heuristic algorithms for full truckloads multi-depot capacitated vehicle routing problem in carrier collaboration," Computers \& Operations Research, vol. 37, no. 5, pp. 950-959, 2010.

[2] S. Hernandez and S. Peeta, "Centralized time dependent multiple-carrier collaboration problem for less-than-truckload carriers," Transportation Research Record: Journal of the Transportation Research Board, vol. 2263, pp. 26-34, 2011.

[3] M. G. Speranza, E. Fernandez, and D. Fontana, "On the collaboration uncapacitated arc routing problem," Computers \& Operations Research, vol. 67, no. C, pp. 120-131, 2015.

[4] Ö. Ergun, G. Kuyzu, and M. Savelsbergh, "Shipper collaboration," Computers \& Operations Research, vol. 34, no. 6, pp. 1551-1560, 2007.

[5] G. Kuyzu, "Lane covering with partner bounds in collaborative truckload transportation procurement," Computers \& Operations Research, vol. 77, pp. 32-43, 2017.

[6] B. Dai and H. Chen, "Mathematical model and solution approach for collaborative logistics in less than truckload transportation," in Proceedings of the International Conference on Computers \& Industrial Engineering, pp. 779-784, IEEE, Troyes, France, July 2009.

[7] B. Dai and H. Chen, "A benders decomposition approach for carrier collaborative planning with less than truckload transportation," International Journal of Modelling in Operations Management, vol. 2, no. 3, pp. 242-265, 2012.

[8] K. Weng and Z. H. Xu, "Flow merging and hub route optimization in collaborative transportation," Journal of Applied Mathematics, vol. 2014, Article ID 621487, 8 pages, 2014.

[9] C. K. Y. Lin, "A cooperative strategy for a vehicle routing problem with pickup and delivery time windows," Computers and Industrial Engineering, vol. 55, no. 4, pp. 766-782, 2008.

[10] R. Sprenger and L. Munch, "A methodology to solve largescale cooperative transportation planning problems," European Journal of Operational Research, vol. 223, no. 3, pp. 626-636, 2012.

[11] C. Quintero-Araujo, A. Gruler, and A. Juan, "Quantifying potential benefits of horizontal cooperation in urban transportation under uncertainty: a simheuristic approach," Proceedings of the Spanish Association for Artificial Intelligence, Springer, Salamanca, Spain, 2016.

[12] O. Yilmazab, "Collaboration among small shippers in a transportation market," European Journal of Operational Research, vol. 218, no. 2, pp. 408-415, 2012.

[13] E. Pérez-Bernabeu, A. A. Juan, J. Faulin, and B. B. Barrios, "Horizontal cooperation in road transportation: a case illustrating savings in distances and greenhouse gas emissions," International Transactions in Operational Research, vol. 22, no. 3, pp. 585-606, 2015.

[14] M. Soysal, J. M. Bloemhof-Ruwaard, R. Haijema, G. A. J. Jack, and V. D. Vorst, "Modeling a green inventory routing problem for perishable products with horizontal collaboration," Computers \& Operations Research, vol. 89, 2017.
[15] K. Fardi, S. Jafarzadeh_Ghoushchi, and A. Hafezalkotob, “An extended robust approach for a cooperative inventory routing problem," Expert Systems with Applications, vol. 116, pp. 310-327, 2019.

[16] N. Mladenovic and P. Hansen, "Variable neighborhood search," Computers \& Operations Research, vol. 24, no. 11, pp. 1097-1100, 1997. 